\title{
Itinéraires Itinéraires
}

Littérature, textes, cultures

2015-2 | 2016

Stumbling blocks. Entraves et obstacles aux circulations

\section{La vue topographique en France au XVIII ${ }^{\mathrm{e}}$ siècle : éclat et mésestime d'un genre}

Veduta in Eighteenth-Century France: A Neglected Genre

\section{Émilie Beck-Saiello}

\section{(2) OpenEdition}

\section{Journals}

Édition électronique

URL : http://journals.openedition.org/itineraires/2819

DOI : 10.4000/itineraires.2819

ISSN : 2427-920X

Éditeur

Pléiade

Référence électronique

Émilie Beck-Saiello, «La vue topographique en France au XVIII siècle : éclat et mésestime d'un genre », Itinéraires [En ligne], 2015-2 | 2016, mis en ligne le 15 février 2016, consulté le 20 avril 2019. URL : http://journals.openedition.org/itineraires/2819; DOI : 10.4000/itineraires.2819

Ce document a été généré automatiquement le 20 avril 2019.

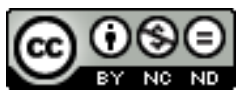

Itinéraires est mis à disposition selon les termes de la licence Creative Commons Attribution - Pas d'Utilisation Commerciale - Pas de Modification 4.0 International. 


\title{
La vue topographique en France au XVIII ${ }^{\mathrm{e}}$ siècle : éclat et mésestime d'un genre
}

\author{
Veduta in Eighteenth-Century France: A Neglected Genre
}

\author{
Émilie Beck-Saiello
}

Tous mes remerciements à Guillaume Glorieux et Jean Boutier, ainsi qu'à Jean-Luc Ryaux et Nicolas Joly pour avoir relu cet article et avoir nourri ma réflexion de leurs remarques et de leurs suggestions.

1 La vue, autrement appelée vue topographique ou, en italien, veduta, est une catégorie de la peinture de paysage qui connut un succès considérable en Europe, en particulier durant la seconde moitié du XVIII ${ }^{\mathrm{e}}$ siècle, au moment de l'essor du phénomène du Grand Tour. Carnevarijs, Van Wittel, Pannini, Canaletto, Guardi, Bellotto, Joli ou encore Hackert ${ }^{1}$ ont ainsi immortalisé les ruines romaines, le Grand Canal à Venise, le golfe de Naples et même Saint Paul de Londres et la Kreuzkirche de Dresde. Qu'en est-il des Français, dont le pays a produit alors de nombreux et célèbres paysagistes, tels Vernet, Hoüel et Hubert Robert $^{2}$ ? Peu d'entre eux ont en fait pratiqué le genre de la vue et appliqué leur talent à la représentation des villes françaises. Peut-on expliquer qu'un des genres artistiques les plus significatifs du XVIII ${ }^{e}$ siècle n'ait connu en France qu'un succès très relatif?

\section{La notion de vue au XVIII ${ }^{\mathrm{e}}$ siècle}

2 L'intérêt pour l'étude de la veduta ne s'est manifesté en France qu'assez tardivement ${ }^{3}$. Le premier auteur à enquêter sur le genre est, en 1966, l'italien Giuliano Briganti, auteur de Gaspar Van Wittel e l'origine della veduta settecentesca, étude fondamentale sur la veduta et sur son fondateur (Briganti 1966). Briganti a également publié en français un ouvrage de divulgation sur le sujet. Il donne pour la première fois une définition précise du genre :

[...] la « veduta » dans son sens le plus courant [est une] vue topographique, c'est-àdire [la] représentation d'un lieu, d'un site caractéristique, d'un monument, ou par 
extension d'un panorama de ville. Il dérive [...] de toute évidence, du terme analogue signifiant " point où tombe la vue ", puis conséquemment aspect d'un lieu tel que le délimite le cadre bien défini de la pyramide visuelle, c'est-à-dire perspective «de paysage » ou de ville, ou d'architecture, ou d'autre chose, incluse dans le plan qui passe à travers le cône de projection de cette pyramide [...]. La veduta est ce paysage décrit avec précision et identifiable, constituant un témoignage « figuré » d'un lieu et d'un milieu déterminés : paysage par conséquent historiquement objectif. (Briganti $1971: 6-7)$ (fig. 1).

Fig. 1. Gaspar Van Wittel, Le Colisée

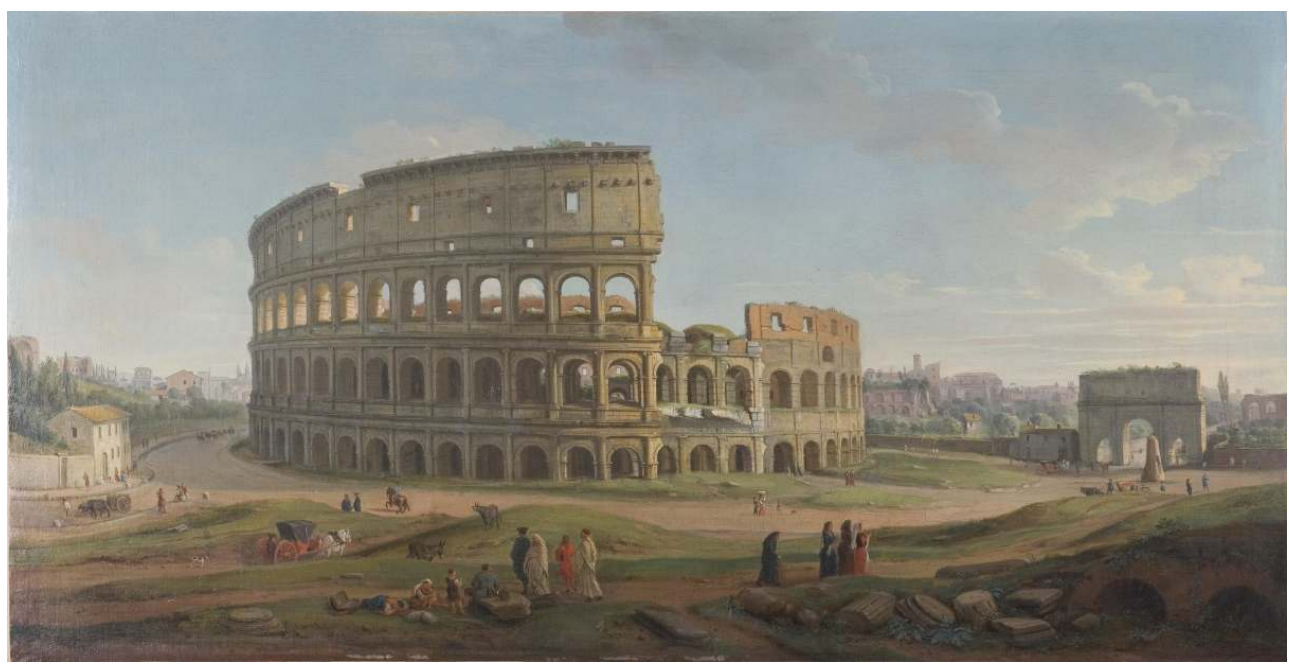

Huile sur toile, environ $100 \times 150 \mathrm{~cm}$.

Courtesy of BNB Art Consulting

Giuliano Briganti en établit également la double origine, nordique et italienne. C'est en Italie et dans l'Allemagne rhénane de la fin $\mathrm{du} \mathrm{XV}^{\mathrm{e}}$ siècle qu'apparaît le " portrait de ville ${ }^{4}$ ». Dans le monde germanique, il est divulgué par les six volumes du Civitates Orbis Terrarum de Braun et Hogenberg (1572-1617). Dans les Flandres, au XVI ${ }^{\mathrm{e}}$ siècle, se développe aussi la "vue topographique ${ }^{5}$ ", sous forme de dessins et de gravures que vont exporter, dans les Provinces-Unies, les artistes protestants ${ }^{6}$.

La pratique de la veduta, c'est-à-dire la représentation en deux dimensions, sur la surface réduite de la toile, d'une large section de paysage (tout ce qui rentre dans le cône visuel), nécessite une bonne connaissance des lois mathématiques et de l'optique ainsi qu'une excellente maîtrise de la perspective et du rapport des proportions. C'est pourquoi la veduta, que l'on appelle au XVII ${ }^{\mathrm{e}}$ et encore au XVIII ${ }^{\mathrm{e}}$ siècle " perspective naturelle ${ }^{7}$ ", doit son développement en Italie aux « peintres de perspectives » qui ont exercé leur talent aussi bien dans les vues architecturales, comme Viviano Codazzi (1604-1670), que dans la scénographie et la quadratura.

5 La rencontre de ces deux traditions, flamande et italienne, s'est faite à Rome à la fin du $\mathrm{XVII}^{\mathrm{e}}$ siècle, lors du séjour qu'y firent alors de nombreux artistes nordiques. Ceux-ci ont peint, dans une veine réaliste, des scènes de rue et des ruines, et réalisé ainsi leurs premières vues souvenirs de la capitale de la chrétienté. Mais celui que l'on considère comme l'un des pères fondateurs du genre (avec Luca Carnevarijs) est le Hollandais Gaspar van Wittel. Il vint en Italie en 1674, tout d'abord pour réaliser des dessins illustrant le cours du Tibre, documents de travail pour l'ingénieur hydraulique Cornelis Meyer, chargé des travaux d'aménagement du fleuve. À partir de 1680, fréquentant les 
ateliers d'impression de guides illustrés de Rome, il s'orienta vers la représentation de vues urbaines et devint rapidement le maître incontesté du védutisme romain de son temps. Il exporta son savoir à Venise (où il séjourna en 1695) et à Naples (il y résida en 1700-1701), exerçant une influence profonde sur les peintres locaux. Rome, Venise et Naples, les trois étapes principales du Grand Tour italien, resteront pendant tout le XVIII siècle les trois foyers de la veduta (Briganti 1966).

6 La veduta est essentiellement commémorative. Elle rappelle le souvenir des lieux visités (fig. 2) ou immortalise un événement (fig. 3). On parle donc de vue souvenir ou de veduta celebrativa (vue de célébration). Elle peut être utilisée pour souligner la prospérité d'une ville ou d'un royaume ainsi que le bon gouvernement du prince et son évergétisme. Mais elle peut être aussi simplement la "photographie » d'une ville, d'un monument ou d'un site, à un moment de son histoire (fig. 4).

Fig. 2. Pierre Jacques Volaire, Éruption du Vésuve vue depuis l'Atrio del Cavallo

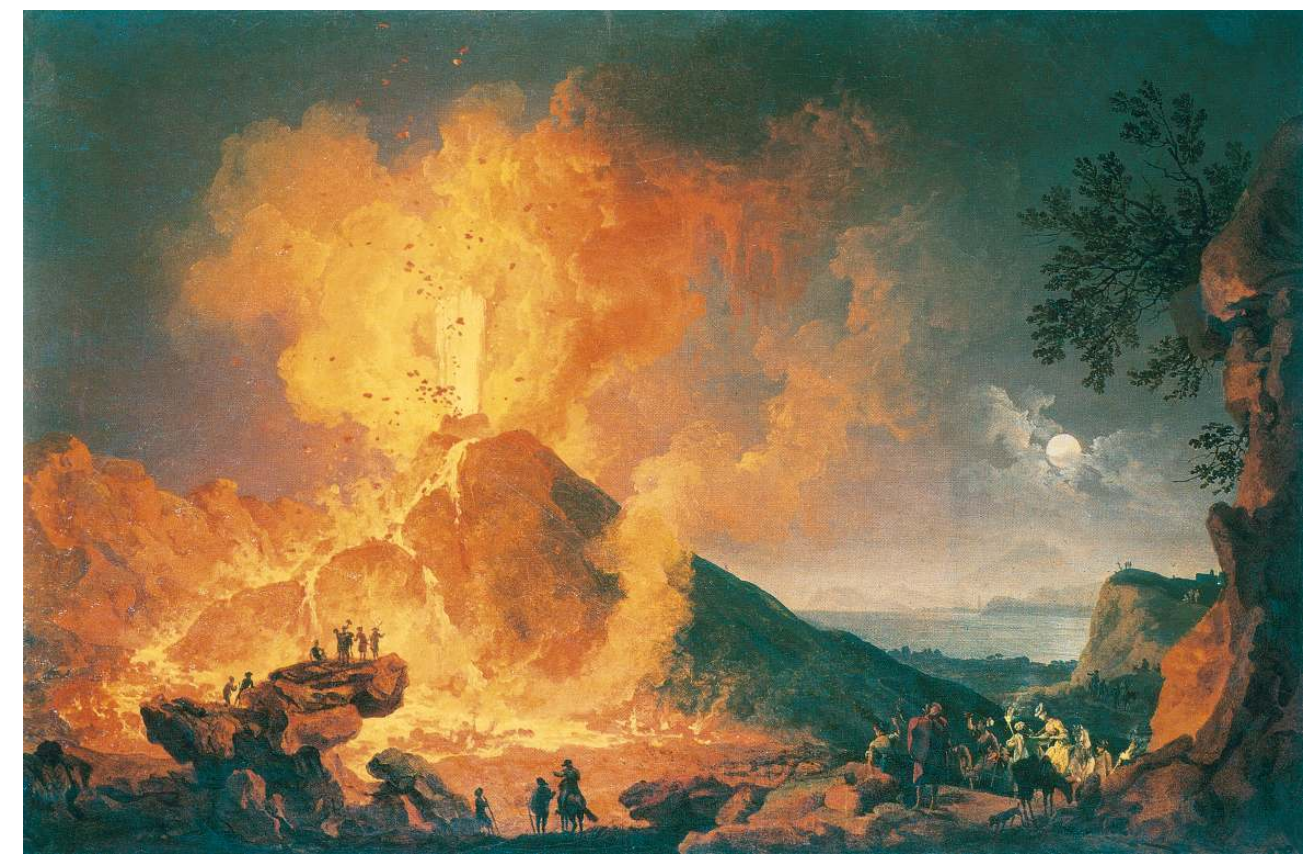

Huile sur toile, $52,5 \times 78,8 \mathrm{~cm}$, après 1771 .

Collection particulière.

Fig. 3. Antonio Joli, Le Cortège royal de Piedigrotta, à Naples

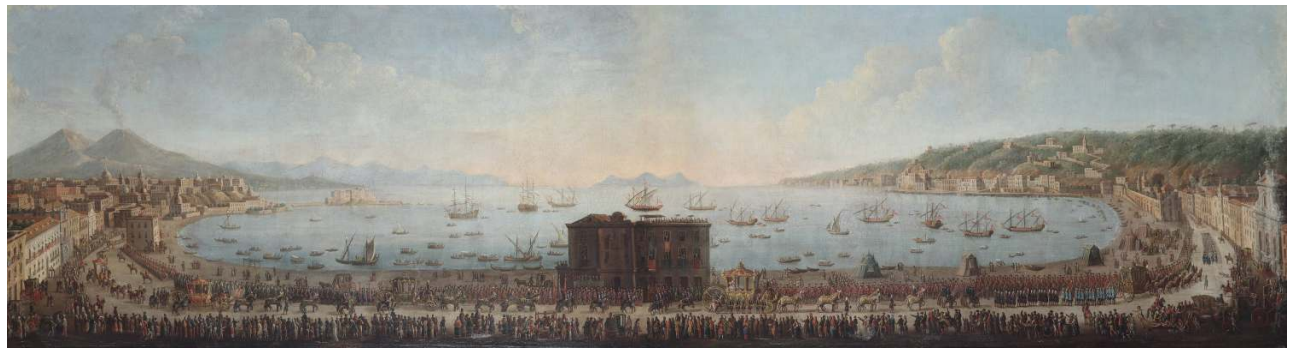

Huile sur toile, $71 \times 255 \mathrm{~cm}$, Vienne, vente Dorotheum, 17 octobre 2012, n 632 .

(c) Dorotheum Vienna, Auction Catalogue 17 $7^{\text {th }}$ October, 2012 
Fig. 4. Pierre-Antoine Demachy, Dégagement de la colonnade du Louvre, vue prise pendant la démolition des vestiges de l'hôtel du Petit Bourbon

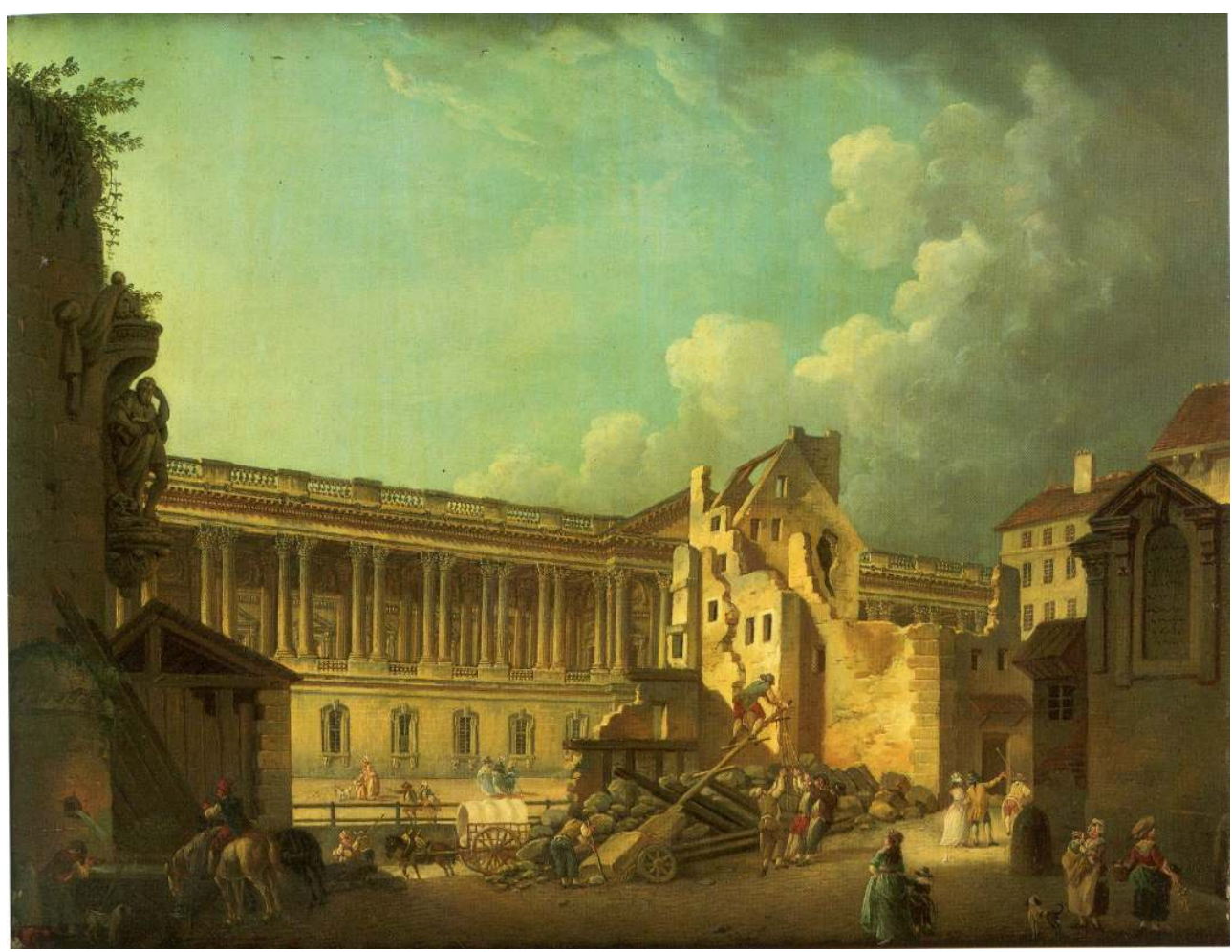

Huile sur bois, $49 \times 64$ cm, signé, Paris, vente Artcurial, 19 décembre 2006, n 58 .

(c) ARTCURIAL BRIEST - POULAIN - F. TAJAN.

L'aristocratie anglaise qui effectue son Grand Tour en est la clientèle principale. L'Angleterre possédait en effet une tradition de vues topographiques (toujours héritée des Flandres), constituées principalement par des paysages portraits représentant les demeures et domaines de l'aristocratie. Une fois en Italie, les voyageurs anglais souhaitaient rapporter une preuve et un souvenir de leur séjour et de leurs expériences. Ces vues sur toile, roulées et transportées dans les bagages ou expédiées par bateau, constituaient l'un des objets privilégiés du marché du Grand Tour.

8 Les Anglais sont les principaux clients des védutistes, mais c'est en réalité toute l'Europe, de la Prusse à la Russie en passant par l'Irlande ou la Suède, qui achète et collectionne des vues. Les œuvres, tout comme les artistes, s'exportent pour satisfaire la demande des amateurs. En revanche, les Français ont peu collectionné le genre de la veduta durant la seconde moitié du xviII ${ }^{e}$ siècle, exception faite d'une petite élite éclairée, que guidait un sentiment national et qu'intéressaient le patrimoine et les transformations urbaines (Guichard 2014, Boutier 2014 : 30-31).

\section{Les raisons d'un relatif insuccès en France}

9 La France a bien été pourtant, à l'époque classique, l'une des grandes patries du paysage. $\mathrm{Au}$ XVIII ${ }^{\mathrm{e}}$ siècle, des artistes comme Joseph Vernet et Hubert Robert ont eu un rayonnement international. Les paysagistes sont alors très nombreux et leur production est fort diversifiée. La majorité d'entre eux a séjourné en Italie et travaillé pour la 
clientèle du Grand Tour. Pourquoi, alors, ont-ils boudé la veduta? Et comment donc qualifier la peinture de paysage qui se pratique en France au Siècle des lumières?

10 Jusqu'au début du xviII ${ }^{\mathrm{e}}$ siècle, on ne distingue essentiellement que deux catégories de paysages : le paysage portrait (ou vue) ${ }^{8}$ et le paysage idéal, jugé plus noble puisqu'il inclut dans la représentation une scène animée et que la nature y a été corrigée par la main du peintre selon les critères du beau idéal ${ }^{9}$. Dans son Cours de peinture par principes, publié en 1708, Roger De Piles introduit une nouvelle catégorie de paysage, le paysage composé ou paysage mixte ${ }^{10}$. Ces trois typologies de paysages sont encore en vigueur lorsque Watelet publie en 1792 l'article «Paysage » dans son Dictionnaire des arts de peinture, sculpture et gravure:

Les aspects que l'on imite fidèlement et tels qu'ils se présentent, s'appellent des vues [...].

Les aspects champêtres, imités en partie d'après la nature et en partie imaginés, sont des paysages mixtes, ou des vues composées.

Les paysages créés sans autre secours que les souvenirs et l'imagination, sont des représentations idéales de la nature champêtre ${ }^{11}$.

11 Le paysage composé ou mixte mêle, comme son nom l'indique, les deux conceptions du paysage (paysage portrait et paysage idéal) et les deux pratiques de l'étude d'après nature et du travail en atelier. Le paysage qu'on a représenté n'est pas vrai mais seulement vraisemblable, puisqu'il présente différents éléments pris dans la réalité mais assemblés par la main du peintre pour créer un effet agréable et pittoresque. Ainsi le Paysage de rivière avec des femmes se baignant dans une cascade de Pierre Jacques Volaire (1729-1799) (collection particulière) qui réunit, dans une même composition, l'église de SainteConstance à Rome, la croix de Pozzano à Castellammare di Stabia, une église romane idéalisée et la grotte du Pausilippe à Naples (fig. 5). 
Fig. 5. Pierre Jacques Volaire, Paysage avec des femmes se baignant près d'une cascade

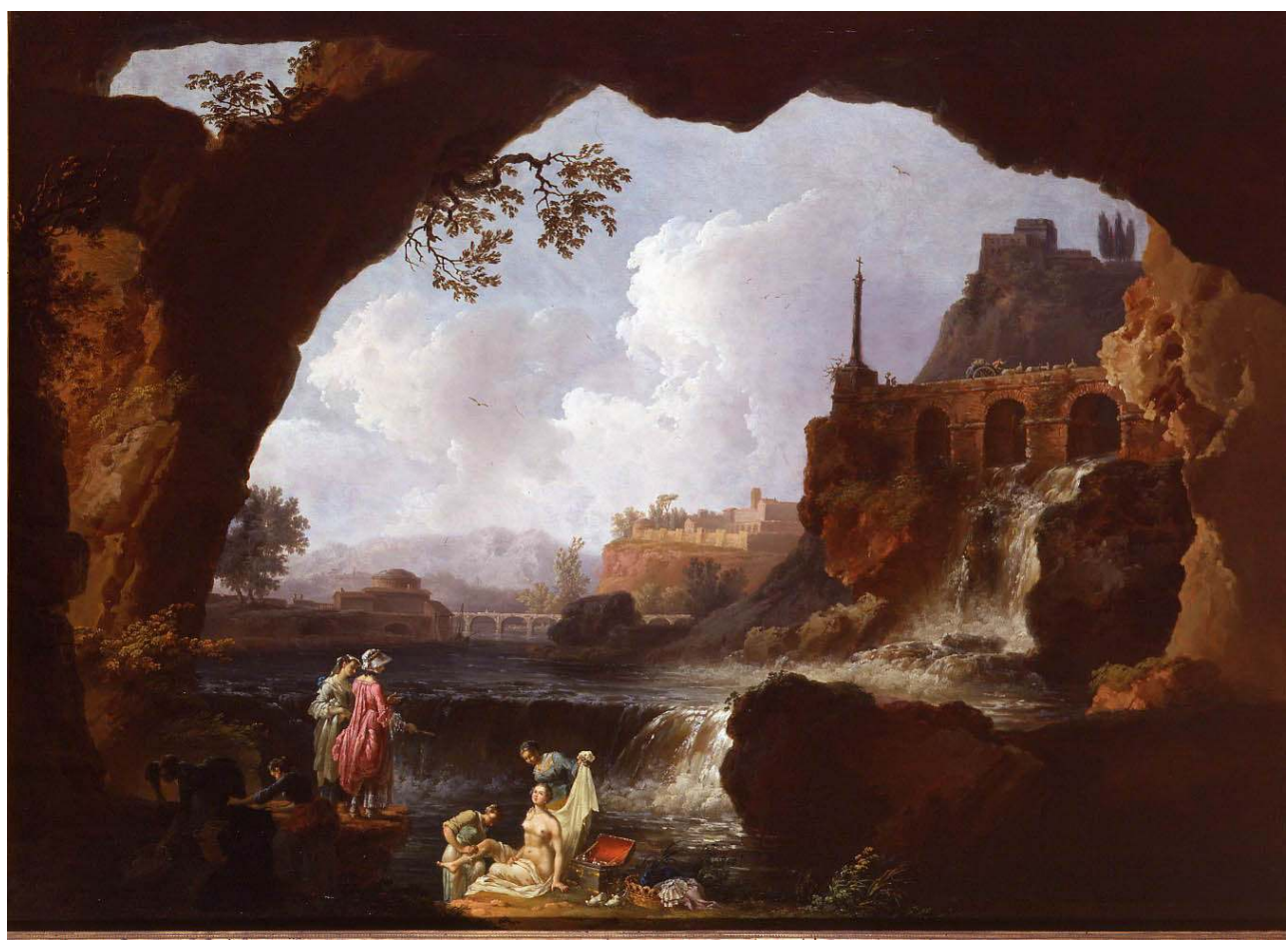

Huile sur toile, 99,7 × 137,8 cm, signé et daté en bas à droite : «Peint par Jacques Volaire à Rome en 1763 », collection particulière.

(c) Galerie Éric Coatalem, Paris.

12 Ce type de paysage a eu en France la préférence de Roger De Piles et des artistes jusque dans les années 1770 . Le courant néoclassique s'accompagne alors d'un retour au paysage idéal et néo-poussinien (voir par exemple François-Xavier Fabre (1766-1837), La Mort de Narcisse, Montpellier, musée Fabre).

Dans ce courant pictural du paysage composé on peut distinguer deux veines ou tendances : une veine pittoresque et une veine narrative.

14 L'abbé Du Bos qualifie de pittoresque « une composition [...] dont le coup d'œil fait un grand effet»([1719] 1993: 90). Charles Coypel, dans une conférence prononcée à l'Académie royale de peinture et de sculpture en 1726, y voit « un choix piquant et singulier des effets de la nature» (Coypel 1726: 54). Le pittoresque implique une préférence pour l'irrégularité, la variété, la complexité, la rudesse ou la difformité, qui peuvent se manifester soit dans les formes de la nature, soit dans le style de l'artiste (fig. 6). 
Fig. 6. Claude-Louis Châtelet, Vue du Val Travers, dans le comté de Neufchâtel

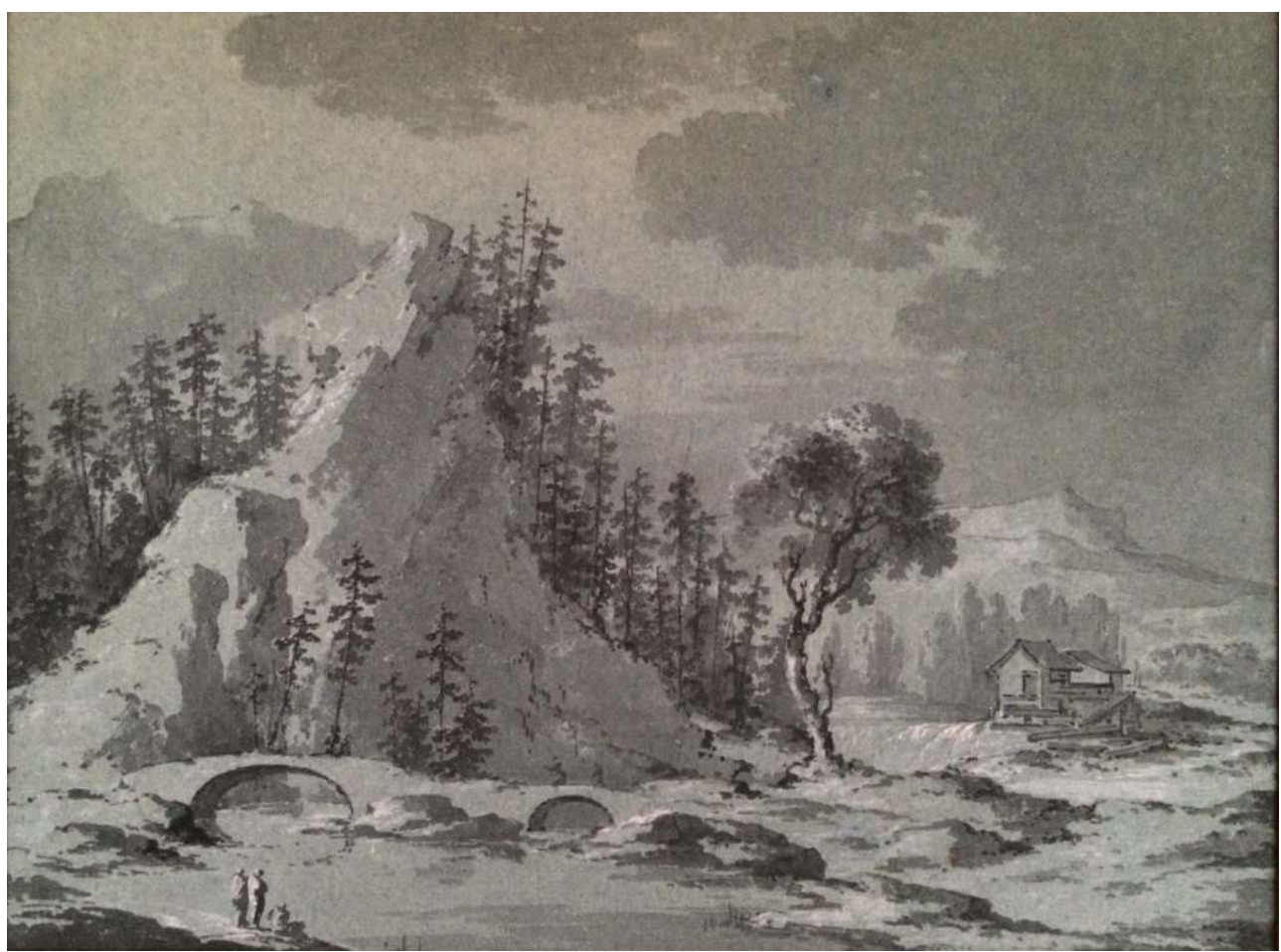

PLUMe ET LAVIS d'ENCRE gRISE ET ReHAUTS DE gOUACHE BLANCHE SUR PAPIER BLEU, 23,5 × 30,8 CM, PARIS, GALERIE FABRICE BONASSO.

(C) Galerie Fabrice Bonasso, Paris.

La veine narrative, en revanche, dans la peinture française de paysage au XVIII ${ }^{e}$ siècle, est ce que Van Mander a appelé en 1604, à propos des paysages flamands du $\mathrm{XVI}^{\mathrm{e}}$ siècle, la " petite histoire ${ }^{12}$ ». Les paysages français ont certes abandonné, au XVIII ${ }^{\mathrm{e}}$ siècle, le récit d'épisodes héroïques tirés de l'histoire, de la littérature ou de la mythologie, mais n'ont pas pour autant renoncé à la description ou au compte rendu d'un événement : naufrage, éruption d'un volcan, tempête, procession, etc. La peinture doit continuer à instruire, les figures doivent nous renseigner sur les mœurs et les coutumes et nous inviter à la réflexion. L'abbé Du Bos écrit à ce propos, en 1719 :

C'est ainsi qu'en ont usé le Poussin, Rubens et d'autres grands maîtres, qui ne se sont pas contentés de mettre dans leurs paysages un homme qui passe son chemin, ou bien une femme qui porte des fruits au marché. Ils y placent ordinairement des figures qui pensent afin de nous donner lieu de penser; ils y mettent des hommes agités de passions afin de réveiller les nôtres et de nous attacher par cette agitation.

(Du Bos 1993 : 18)

16 On ne peut pas dire pour autant que la vue soit absente de la production des peintres et dessinateurs de paysages au XVIII ${ }^{e}$ siècle. Les deux commandes royales passées par Louis XV à Vernet pour la série des Ports de France, et par Louis XVI à Hubert Robert pour celle des monuments antiques de Provence, en sont des exemples célèbres ${ }^{13}$ (et il faut $\mathrm{y}$ ajouter la Vue d'Avignon de Vernet, récemment acquise par le musée du Louvre). La série des Ports de Vernet (comme celle de La Rochelle, fig. 7) sont ainsi des portraits assez fidèles des villes portuaires françaises, où le souci du détail vrai (embarcations, costumes, marchandises, etc.) ne nuit pas à la vision d'ensemble ${ }^{14}$. Il existe par ailleurs un courant (plus qu'un genre) de vues de villes, qui prend sa source dans les vignettes placées aux 
marges des cartes urbaines ${ }^{15}$. Au XVII ${ }^{\mathrm{e}}$ siècle, ce sont des artistes flamands et hollandais qui s'illustrent dans le portrait de ville, tant en dessin qu'en peinture: Abraham de Verwer (1585-1650), Pieter Wouwerman (1623-1682), Reinier Nooms, dit Zeeman (vers 1623 - avant 1667), ou Lievin Cruyl (1634 - avant 1720). À ces noms il faut ajouter, pour le deuxième quart $\mathrm{du} \mathrm{XVIII}^{\mathrm{e}}$ siècle, celui du peintre Charles Léopold Grevenbroeck (actif à Paris en 1730 et à Naples après 1758/59) et dont l'œuvre majeure est la commande passée en 1738 par le surintendant Philibert Orry des quatre vues de Paris, exposées au Salon de $1741^{16}$. La Vue des hauteurs de Belleville du musée Carnavalet, à titre d'exemple, est une minutieuse vue topographique au dessin subtil, préparée avec une rigueur scientifique par de nombreux repérages et études. À partir de la seconde moitié du XviII siècle, quelques peintres de paysage français, comme Alexandre Noël (1752-1834), s'intéressent à ce type de représentations ${ }^{17}$. Mais c'est Nicolas Jean-Baptiste Raguenet (1715-1793), puis Pierre-Antoine Demachy (1723-1807) ${ }^{18}$, qui se feront une spécialité dans les vues urbaines et particulièrement de celles de Paris. Le cas de Demachy est probablement l'exemple qui confirme la règle : il fit une brillante carrière académique, ses œuvres remportèrent un réel succès et furent collectionnées par des amateurs distingués. Mais il mourut pauvre, n'eut guère de descendance artistique et ses tableaux furent souvent âprement critiqués lors de leur présentation au Salon.

Fig. 7. Joseph Vernet, Vue du port de La Rochelle

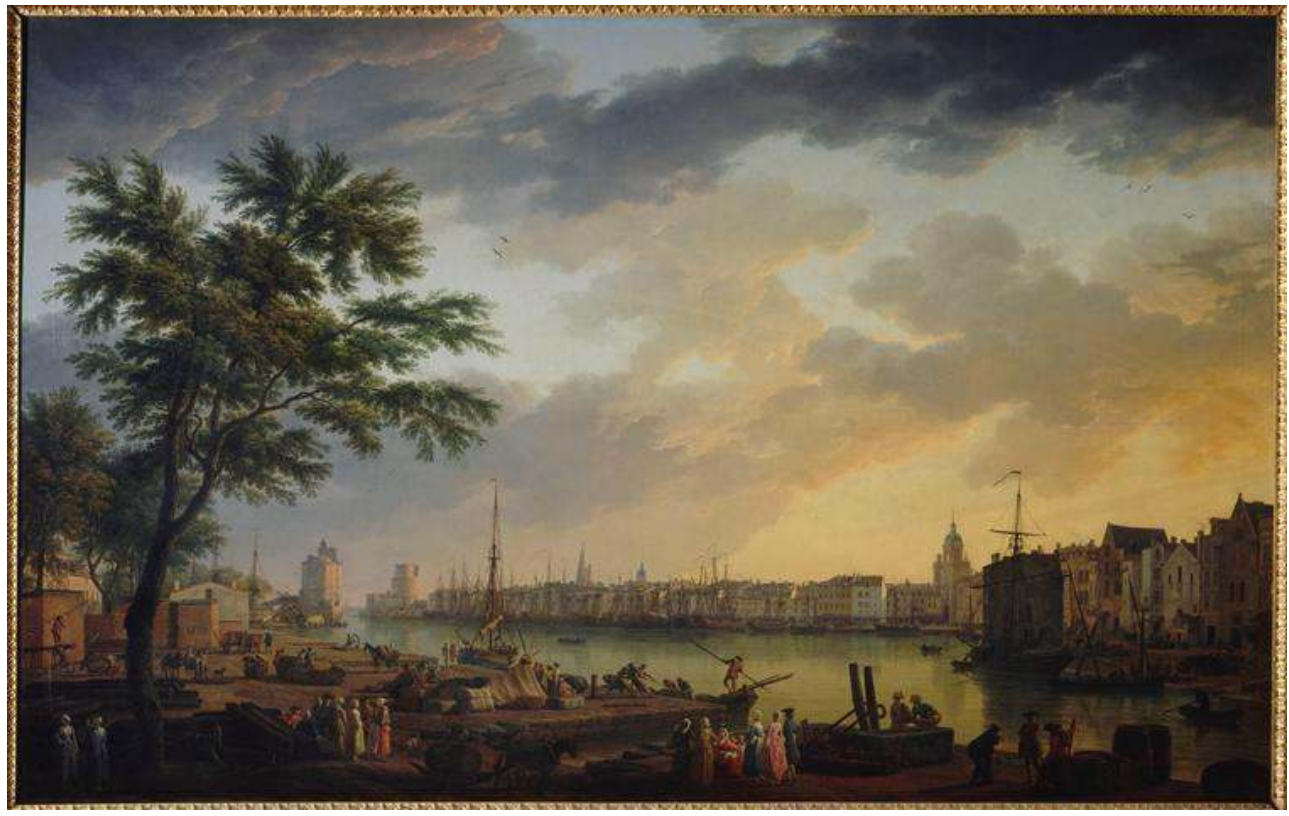

Huile sur toile, $165 \times 203$ cm, signé et daté en bas à droite : «Peint à La Rochelle par J. Vernet en $1762 »$, Paris, musée du Louvre.

(c) RMN.

Parallèlement à la production de vues peintes se développèrent de nombreuses vues dessinées, réalisées, en France et en Europe, par des artistes comme Jean-Baptiste Lallemand (1716-1803 ?), Jean Hoüel, Claude-Louis Châtelet (1749/50-1795, fig. 8), ou encore par des architectes de formation, comme Charles-Louis Clérisseau (1721-1820), Louis-Jean Desprez (1743-1804) et Victor-Jean Nicolle (1754-1826, fig. 9). Celles-ci constituent souvent la documentation illustrée d'un voyage fait en France ou dans des 
contrées plus lointaines et elles sont destinées à un public de curieux. Nous reviendrons sur ces exceptions.

Fig. 8. Claude-Louis Châtelet, Vue de Catanzaro

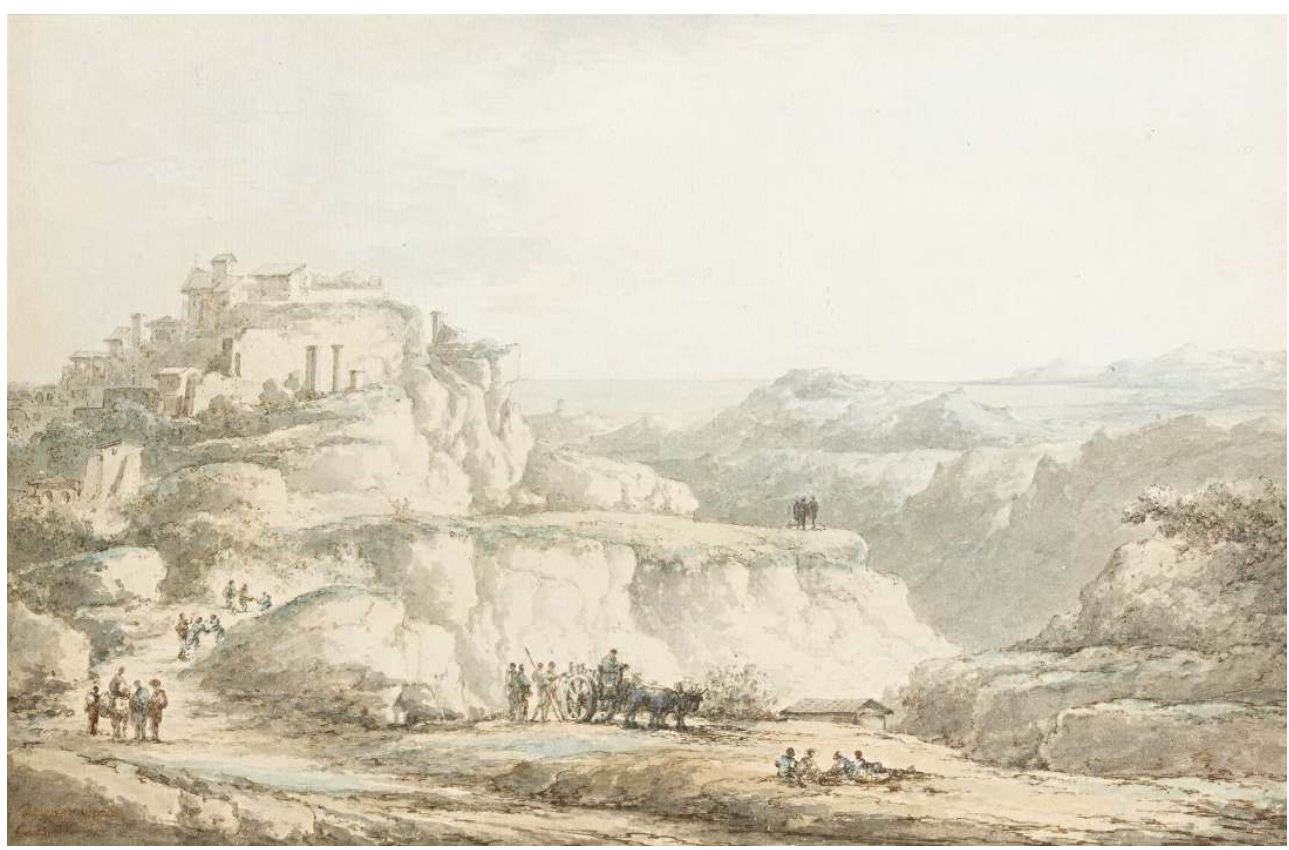

PLUMe et ENCRE NOIRE ET gRISE, AQUARELLE, 16,5 × 25, 5 CM, SIgNÉ AU VERSO : " CHÂTELET DeLIN. " COURTESY OF NICOLAS JOLY. 
Fig. 9. Victor-Jean Nicolle, Vue des restes de l'Hippodrome de Constantin ou du Temple de Bacchus à Rome

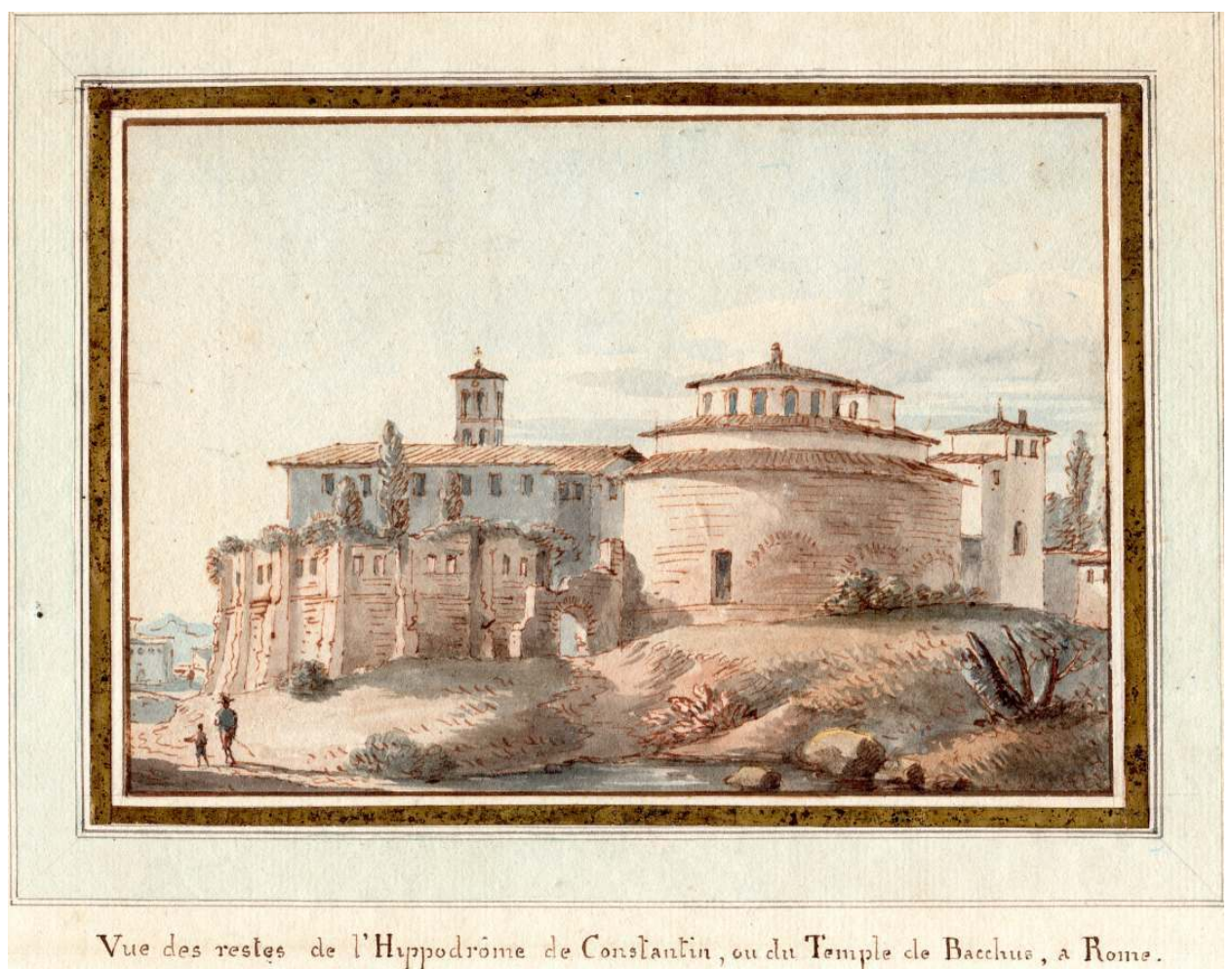

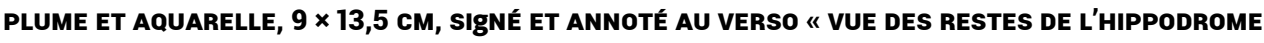
de CONStANTIN OU du temple de baCChus À ROME ", PARIS, GALERIE fabrice bonasso.

Courtesy of Jean-Luc Ryaux.

Il est temps désormais d'analyser les raisons du peu de succès que connut la veduta en France au XVIII ${ }^{\mathrm{e}}$ siècle, comparativement aux autres pays européens. La France n'a pas, à la différence des Flandres ou de l'Angleterre, une forte tradition de vues topographiques (ou paysages portraits). Par ailleurs, les différentes pratiques artistiques qui sont à l'origine du développement de la veduta en Italie - les vues architecturales, l'illusion scénographique et le quadraturisme - n'ont pas connu de large développement en France. Un art classique fait de raison, de règles, de symétrie et d'équilibre y a prévalu à partir de Louis XIV et a été préféré à un art de l'illusion.

On remarquera aussi que, dès l'institution de la surintendance des Bâtiments du roi et la création des académies $^{19}$, la production des artistes, architectes, cartographes, topographes, décorateurs ou ingénieurs s'est trouvée codifiée et cantonnée à un secteur d'activité. Si l'on excepte les petits maitres travaillant pour des commandes privées ou municipales, et contraints par la nécessité à réaliser des plans, des relevés, des décors éphémères pour des événements de l'histoire locale, la plupart des artistes assumèrent une fonction bien particulière. C'est ainsi qu'Adam François Van der Meulen (1632-1690) et Jean-Baptiste Martin (1659-1735) furent peintres des batailles de Louis XIV; Pierre Denis Martin, "peintre ordinaire du roi », se spécialisa dans la représentation des châteaux royaux; Louis Nicolas de Lespinasse (1734-1808) enseigna la tactique de la topographie à l'École royale militaire; Jean-Baptiste Génillion (1750-1829) et Nicolas Ozanne (1728-1811) furent respectivement peintre officiel et dessinateur de la Marine et Laurent-Antoine Volaire (1723/24, documenté jusqu'en 1766), pilote du roi et dessinateur- 
géographe. Ce mélange des genres (scénographie, topographie, cartographie, peinture de paysage, etc.), qui a été si favorable au développement et à l'essor de la veduta en Europe, ne s'est guère opéré en France (si l'on excepte le cas de Demachy qui fut formé par l'Italien Giovanni Niccolò Servandoni (1695-1766)). Il est vrai également que certains des artistes précédemment mentionnés figurent parfois dans les documents comme peintres de vues - c'est le cas de Génillion (fig. 10) et de Louis-Nicolas Van Blarenberghe (1716-1794) - mais leurs vues constituent essentiellement une documentation à des fins militaires. Nous reviendrons sur le sujet.

Fig. 10. Jean-Baptiste Génillion, Éruption du Vésuve

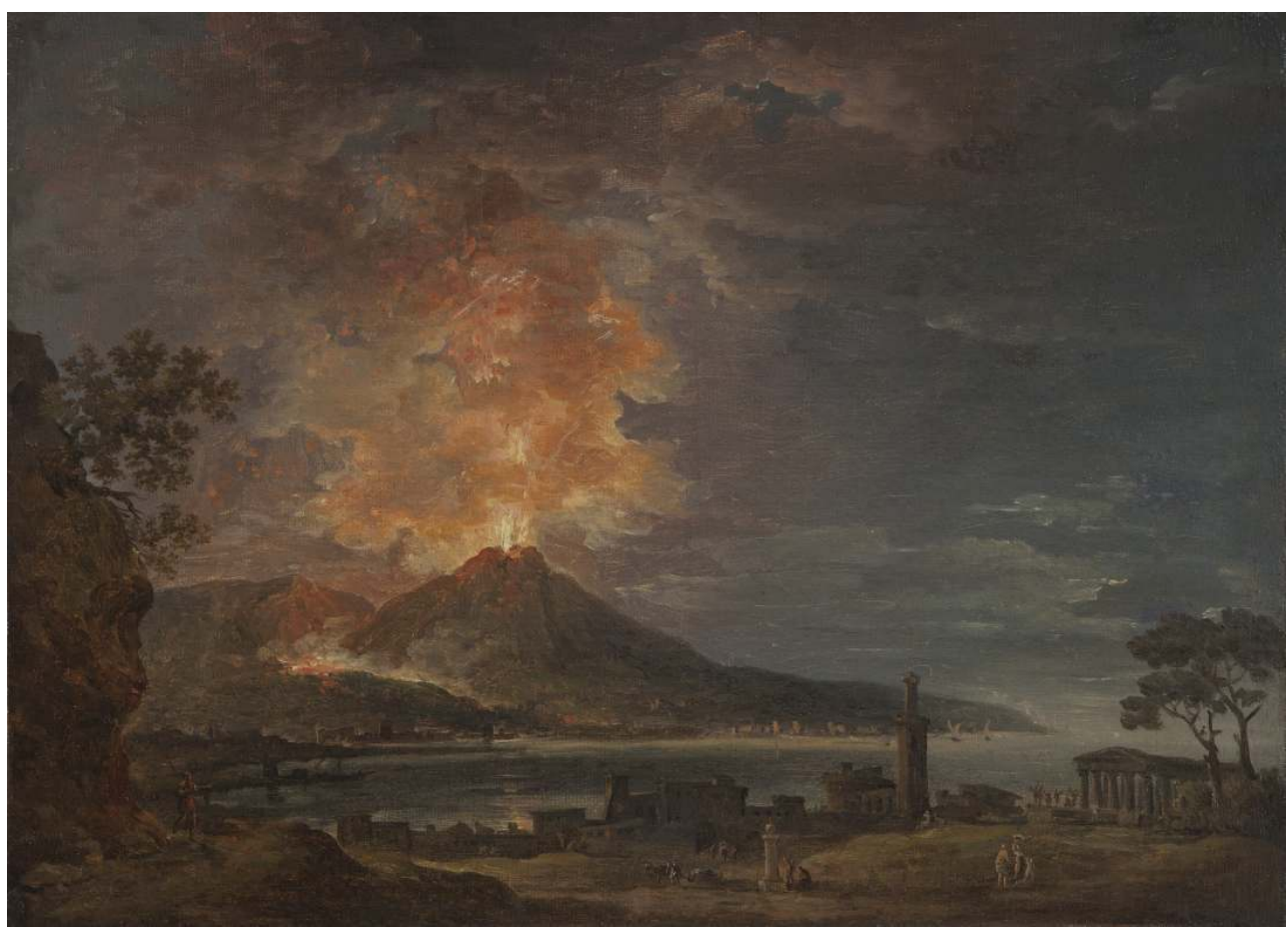

Huile sur toile, 34,5 × 38,2 cm, signé en bas à gauche : «Genillion ».

Collection particulière.

Il existe de plus, en France, une très forte hiérarchie des genres picturaux, établie et maintenue par l'Académie. Le paysage et la nature morte se situent en bas de l'échelle, après la peinture d'histoire, le portrait et la scène de genre. En dépit du succès de certains grands artistes comme Vernet et Hubert Robert, le développement de la pratique du paysage chez de nombreux peintres, malgré encore la faveur dont jouit le genre auprès du public et enfin, malgré les idées pionnières de De Piles et de Cochin, le paysage reste considéré pendant tout le XVIII ${ }^{\mathrm{e}}$ siècle comme un genre mineur. L'on pense en effet que, dans le paysage, c'est l'imitation et donc l'habileté manuelle qui prévalent sur l'imagination et l'invention, facultés qui avaient conduit les peintres à hisser leur activité au rang d'art noble, d'art de la pensée, au même titre que les arts libéraux (Harent 2005 : $214)^{20}$. Au sein du paysage existaient, comme nous l'avons vu, différentes catégories qui, elles aussi, étaient soumises à une hiérarchie. Ainsi Watelet plaçait à son sommet les représentations idéales, suivies par les paysages composés, et reléguait les vues en bas de l'échelle; ceci, en accord avec la tradition académique qui assignait une valeur majeure à l'imagination par rapport à la représentation fidèle de la réalité. Cette hiérarchie fut 
même reprise par un peintre de paysage, Pierre-Henri de Valenciennes (1750-1819), qui rédigea un traité sur le genre en 1800, dans lequel il écrivit :

Le genre du paysage portrait n'exige pas, à la rigueur, beaucoup de génie. Il n’y a que les yeux et la main qui travaillent [...].

Le paysage héroïque [est le] genre le plus noble de tous et qu'on ne saurait traiter sans avoir beaucoup de génie. (Valenciennes $1800: 479,483$ )

Valenciennes sera d'ailleurs, à la fin du XVIII ${ }^{\mathrm{e}}$ siècle, à l'origine du renouveau du paysage héroïque ou idéal, qui prendra au XIX ${ }^{\mathrm{e}}$ siècle le nom de paysage historique.

Ouvrons une brève parenthèse. Dans la catégorie du paysage portrait figurent les nombreuses vues dessinées, aux fins d'être publiées dans les différents Voyages ou Tableaux pittoresques. Celles-ci furent considérées par les critiques comme des œuvres avant tout documentaires, des illustrations, au même titre que celles de l'Encyclopédie. Et ce, malgré leur indéniable qualité formelle.

Si les artistes n'ont pas pratiqué le paysage héroïque ou idéal avant les années 1770-1780, ils ont eu soin néanmoins, comme nous l'avons vu, d'introduire dans leurs paysages un contenu narratif et de raconter la vie de leur temps. Ce fut le cas de Vernet par exemple, qui sut représenter, dans sa série des Ports de France comme dans ses vues de Rome ou de Naples, les mœurs, les coutumes et les costumes des habitants des villes et des marins et commerçants étrangers, et raconter l'histoire d'une société et de son mode de vie. L'abbé de La Porte, dans sa critique du Salon de 1755, écrit à propos de deux de ses tableaux :

Sur son port de Marseille et dans son arsenal de Toulon, dont les détails sont exacts, sans confusion, et méthodiquement ordonnés, on apprendroit des choses que bien des personnes devroient sçavoir, et dont on auroit de la peine à se faire instruire. Pourquoi nos peintres, presque toujours préoccupés d'idées vagues, singulières, et bien souvent inutiles, ne s'asservissent-ils pas quelquefois à représenter des choses connues de nos jours, mais toujours d'un beau choix? Ils seroient précieux aux étrangers, à la postérité. On liroit dans leurs tableaux l'histoire des coutumes, des arts, des nations ; ils seroient toujours intéressants, s'ils étoient vrais, parce qu'ils seroient utiles. (La Porte 1755 : 12-13)

Le faible développement de la veduta en France par rapport aux autres pays européens s'explique aussi par l'organisation politique et culturelle de chaque nation, comme l'a mis en évidence Jean Boutier :

La centralisation française des activités intellectuelles et artistiques est sans aucun doute un incroyable levier pour imposer ces hiérarchies, sans qu'il puisse y avoir le développement de productions alternatives. Les commandes locales éventuelles renvoient toujours aux hiérarchies officielles, car les villes elles aussi sont prises dans le système royal et la vue ne semble pas avoir été un moyen pour mettre en avant l'orgueil citadin (que de toute façon la monarchie n'apprécierait guère) ${ }^{21}$.

En Italie, le polycentrisme artistique et le fort campanilisme ${ }^{22}$ ont encouragé le développement de l'iconographie urbaine.

Quant à l'Angleterre, malgré le poids de Londres, elle n'est pas aussi politiquement centralisée que la France: la cour de Londres n'a pas l'importance de celle de Versailles, et ne dicte pas de choix culturels aussi forts. On ne peut certes négliger le rôle de la Royal Academy, mais il y a en Angleterre plus de diversité qu'en France. La vie provinciale y est extrêmement forte, avec sa culture propre. Le pays souffre aussi d'un sentiment de faible légitimité artistique, au moins jusque dans les années $1770^{23}$.

Enfin, on pourra observer que la demande de vues topographiques a émané principalement de pays de religion et de culture réformées: Provinces-Unies au XVII ${ }^{\mathrm{e}}$ 
siècle, puis Grande-Bretagne au XVIII et, dans une moindre mesure, pays germaniques. Pourquoi ? Parce que le paysage portrait - comme d'ailleurs le portrait qui a connu dans ces mêmes pays un large développement - est une photographie de la réussite sociale. Il fixe le statut ou l'ascension sociale d'un homme ou d'une famille. Les vues topographiques des demeures anglaises et de leurs domaines en sont bien évidemment l'exemple le plus éloquent, mais les vues souvenirs des villes visitées pendant le Grand Tour le sont également. Elles sont la preuve que leurs propriétaires appartiennent à cette élite fortunée des voyageurs qui a parcouru l'Italie, complété sa formation et ses connaissances au contact de sociétés différentes, et prouvé, par l'acquisition de tableaux, un goût prononcé pour la culture et les arts.

En France, les seules véritables vues ont été celles des ports de France et des monuments antiques de Provence commandées par Louis XV et Louis XVI, ainsi que les vues de Paris de Demachy, acquises par les cercles proches de l'Académie. CEuvres de propagande, elles devaient montrer la force de la marine militaire et commerciale et le dynamisme de la politique édilitaire, souligner l'unité du royaume ou bien décrire les richesses artistiques d'un pays qui n'avait plus rien à envier à l'Italie. Les autres vues sont les scènes de batailles de Van der Meulen ou de Martin. Mais peut-on parler de vues? Les scènes de bataille ou les tableaux d'histoire (et d'histoire contemporaine) constituent aussi un genre de peinture autonome depuis le XVII ${ }^{\mathrm{e}}$ siècle. S'il y eut des vues topographiques en France, au XVIII ${ }^{e}$ siècle, elles ne furent pas créées à l'initiative d'une classe sociale et pour illustrer son statut et sa réussite, mais pour fixer les grands événements de l'histoire nationale et ses grands succès dans le domaine maritime, commercial ou artistique.

En guise de conclusion, je souhaiterais revenir sur un passage du traité de Valenciennes, Élémens de perspective pratique à l'usage des artistes, évoqué plus haut. Dans ce traité le paysage composé, type de paysage le plus pratiqué par les Français au XviII siècle, semble à première vue totalement absent. Mais on le trouve en fait inclus dans le paragraphe consacré au paysage portrait. Selon Valenciennes, le paysage portrait est constitué par la représentation objective de la nature telle qu'elle apparait dans le cône visuel, ou bien par la représentation composite d'éléments variés, pris en différents lieux. Selon lui, en effet, le paysage, tel qu'il se présente à notre regard, est rarement parfait; il faut par conséquent en sélectionner les éléments les plus beaux et les regrouper dans une même composition (ce qui revient donc à réaliser un paysage composé). Ainsi, pour Valenciennes, la vue topographique au sens strict, représentation fidèle d'un lieu, n'existe pas. De fait, Giuliano Briganti avait déjà mis en évidence la liberté que prenaient parfois les védutistes avec la réalité. André Corboz est allé plus loin, montrant que chez Canaletto, le plus célèbre védutiste du XvIII ${ }^{e}$ siècle, il n'y avait pas de limites entre la veduta et le caprice ou paysage imaginaire (Corboz 1985). Pour autant, on ne pourra néanmoins aller jusqu'à nier l'existence, au xvIII siècle, des vues topographiques produites, comme nous l'avons souligné, majoritairement en Italie et pour une clientèle principalement anglaise et protestante. À ce courant réaliste du paysage, la France aura finalement préféré une version simplement naturaliste, au contenu narratif. 
Fig. 11. Pierre-Henri de Valenciennes, L'Ancien Jeu de l'outre

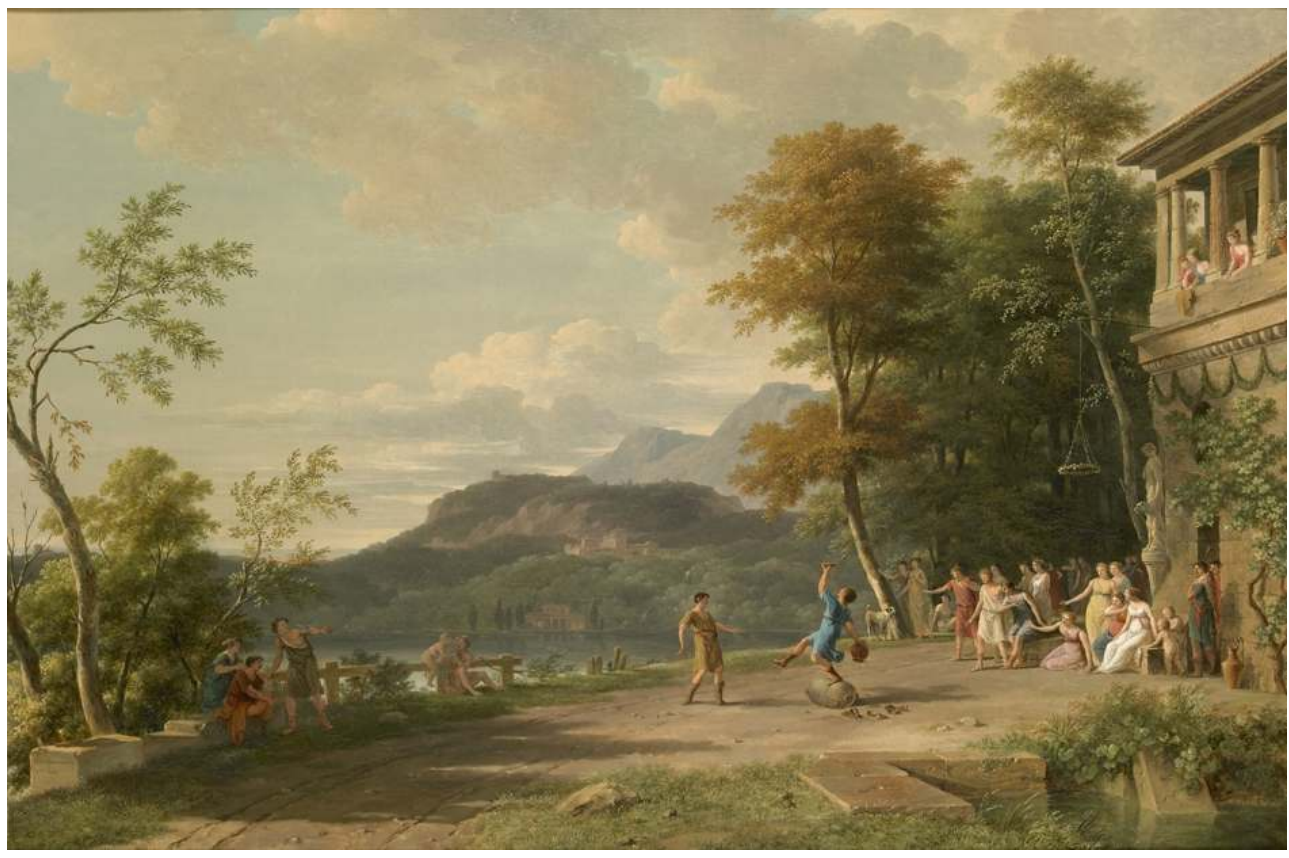

Huile sur toile, 65,8 × 97,5 cm, signé et daté en bas à gauche : «P. Valenciennes / an XII », Collection particulière.

(c) Courtesy of Galerie Jean-François Heim, Bâle.

\section{BIBLIOGRAPHIE}

Boutier, Jean, 2014, « Pittura topografica e vedutismo nelle città della Francia dei Lumi ", dans C. De Seta (dir.), L'immagine della città europea, dal Rinascimento al Secolo dei Lumi, cat. exp. (Venise, Musée Correr, 8 février - 18 mai 2014), Milan, Skira, p. 22-39.

Braun, Georg et Hogenberg, Frans, 1572-1617, Civitates Orbis Terrarum, Cologne, typis Theod. Graminoei, 6 vol.

Briganti, Giuliano, 1966, Gaspar Van Wittel e l'origine della veduta settecentesca, Rome, Bozzi.

Briganti, Giuliano (dir.), 1968, L'Europa dei vedutisti, Trieste, RAS.

Briganti, Guliano, 1969, I vedutisti, Milan, Bompiani-Electa (trad. fr., Les Peintres de vedute, Paris, Electa France, 1971).

Cayeux, Jean de, 1997, Le Paysage en France : de 1750 à 1815, Saint-Rémy-en-l'Eau, Monelle Hayot. Corboz, André, 1985, Canaletto : una Venezia immaginaria, Milan, Alfieri Electa.

Coypel, Charles, 1726, Discours prononcés dans les conférences de l'Académie royale de peinture et de sculpture par M. Coypel..., Paris, Jacques Collombat.

De Seta, Cesare, 2011, Ritratti di città. Dal Rinascimento al secolo XVIII, Turin, Einaudi. 
Dorbec, Prosper, 1908, « Les premiers peintres du paysage parisien », Gazette des Beaux-Arts, $3^{\mathrm{e}}$ période, CIII, p. 441-470.

Du Bos, Jean-Baptiste, [1719] 1993, Réflexions critiques sur la poésie et la peinture, Paris, École nationale supérieure des beaux-arts.

Guichard, Charlotte, 2014, « Les amateurs au XVIII ${ }^{\mathrm{e}}$ siècle », dans F. Roussel-Leriche et M. Petkowska Le Roux (dir.), Le Témoin méconnu : Pierre-Antoine Demachy, cat. exp. (Versailles, Musée Lambinet, 15 février - 18 mai 2014), Paris, Magellan \& Cie, p. 42-47.

Guiffrey, Jules, 1893, « Correspondance de Joseph Vernet avec le Directeur des Bâtiments sur la collection des Ports de France, et avec d'autres personnes sur divers objets, 1756-87 », Nouvelles archives de l'art français, $3^{\mathrm{e}}$ série, vol. 9, p. 1-99.

Harent, Sophie, 2005, « Peindre la ville au XVIII ${ }^{\mathrm{e}}$ siècle », dans A. Gady et J.-M. Pérouse de Montclos (dir.), De l'esprit des villes. Nancy et l'Europe urbaine au siècle des Lumières, 1720-1770, cat. exp. (Nancy, Musée des beaux-arts, 7 mai-22 août 2005), Versailles, Artlys, p. 206-215.

La Porte, Joseph de, 1755, Sentiments sur plusieurs des tableaux exposés cette année dans le grand salon du Louvre, s. 1.

Lomazzo, Giovanni Paolo, 1584, Trattato dell'arte della pittura, scoltura et architettura, Milan, Paolo Gottardo Pontio.

Manœuvre, Laurent et Reith, Éric, 1994, Joseph Vernet 1714-1789 : Les Ports de France, Arcueil, Anthèse.

Schattborn, Peter, 1994, « La naissance du paysage naturaliste aux Pays-Bas et l'influence de la topographie aux environs de 1600 », dans C. Legrand, J.-F. Méjanès et E. Starcky (dir.), Le Paysage en Europe du XVI $I^{e}$ au XVIII siècle, actes du colloque (Paris, musée du Louvre, 25-27 janvier 1990), Paris, Réunion des musées nationaux.

Troili, Giulio, 1683, Paradossi per pratticare la prospective senza saperla, Bologne, Per Gioseffo Longhi, [En ligne], http://gallica.bnf.fr/ark:/12148/btv1b55005650r.

Lanzi, Luigi, 1795-1796, Storia pittorica dell'Italia, Bassano, Remondino.

Valenciennes, Pierre-Henri de, an VIII (1800), Élémens de perspective pratique à l'usage des artistes, suivis de réflexions et conseils à un élève, Paris, Desenne.

Van Mander, Karel, 1604, Het Schilder-boeck, Haarlem, Passchier Wesbusch.

Watelet, Claude-Henri et Lévesque, Pierre Charles, [1788] 1792, Encyclopédie méthodique, $2^{\mathrm{e}}$ éd. Dictionnaire des arts de peinture, sculpture et gravure, Paris, Prault.

\section{NOTES}

1. Luca Carnevarijs (1663-1730), Gaspar van Wittel (1653-1736), Giovanni Paolo Pannini (1691-1765), Giovanni Antonio Canaletto (1697-1768), Francesco Guardi (1712-1793), Bernardo Bellotto (1722-1780), Antonio Joli (vers 1700-1777) et Jacob Philipp Hackert (1737-1807).

2. Joseph Vernet (1714-1789), Jean Hoüel (1735-1813) et Hubert Robert (1733-1808).

3. Si l'on excepte l'article de Prosper Dorbec, publié dès 1908, et qui s'intéresse essentiellement aux vues des environs de Paris. Il convient de citer, pour l'étude générale du védutisme en France, l'article de fond de Jean Boutier (2014, avec la bibliographie sur les différents représentants du « genre »), ainsi que Sophie Harent (2005). 
4. Le terme apparaît pour la première fois vers 1555 sur une petite vue de Sienne à l'eau-forte, Il vero ritratto della città di Siena. Le portrait de ville vise à restituer l'image de la cité dans sa splendeur, et documente la morphologie du site, la structure urbanistique et l'architecture de la ville. Voir Cesare De Seta (2011, en particulier introduction et chap. I).

5. La vue topographique est une représentation fidèle d'une ville ou d'un site réalisée d'après nature. La vue tout court peut s'attacher en revanche à la description d'éléments de paysage (cabane, rochers, etc.). Voir Claude-Henri Watelet et Pierre Charles Lévesque ([1788] 1792, V : 832).

6. Sur l'origine et le développement du portrait de ville, voir Cesare De Seta (2011).

7. La perspective naturelle est un terme d'optique ; elle se réfère, depuis le $\mathrm{XV}^{\mathrm{e}}$ siècle à la vision humaine, à la vision directe, et s'oppose à la perspective artificielle ou mathématique dont les principes furent exposés par Filippo Brunelleschi. Comme l'a rappelé Giuliano Briganti ([1969] 1971: 7), le terme de perspective ou perspective naturelle, utilisé par exemple par Lomazzo ( Trattato dell'arte della pittura, scoltura et architettura, 1584), devient synonyme de veduta sous la plume de Giulio Troili, auteur, en 1683, des Paradossi per pratticare la prospective senza saperla. L'usage du mot " perspective » pour désigner le genre de la veduta perdurera néanmoins pendant tout le $\mathrm{XVIII}^{\mathrm{e}}$ siècle (on le retrouve encore dans la Storia pittorica dell'Italia de Luigi Lanzi, publiée en 1796).

8. Voir Pierre-Denis Martin (1663-1742), Vue du château de Chambord du côté du parc, Versailles, musée national des châteaux de Versailles et de Trianon. Martin travailla avec son frère JeanBaptiste, dit Martin des Batailles (1659-1735), qui avait lui-même collaboré avec Adam François van der Meulen (1632-1690) comme peintre de batailles. Ce genre des batailles constitue une autre forme de veduta qui se fonde sur le dessin topographique des places assiégées. La bibliographie à ce sujet est très vaste et la filiation avec la pratique de la veduta est forte, tant dans l'œuvre de Pierre-Denis Martin que dans celle de Jean-Baptiste. Le travail des védutistes français au XVIII ${ }^{\mathrm{e}}$ siècle est ainsi à mettre en relation avec la pratique du "portrait au naturel » des villes tel qu'il était pratiqué au XVI ${ }^{\mathrm{e}}$ siècle et en France au XVII ${ }^{\mathrm{e}}$ siècle.

9. Voir par exemple Nicolas Poussin (1594-1665), Le printemps, dit aussi Adam et Ève au paradis terrestre, Paris, musée du Louvre.

10. Voir par exemple Adrien Manglard (1695-1760), Paysage fantastique avec la tombe de Cecilia Metella, localisation inconnue.

11. Claude-Henri Watelet («Paysage », [1788] 1792, IV : 9). Watelet, qui apprécie néanmoins les vues, leur consacre également une entrée spécifique dans son Dictionnaire (Ibid., V : 832).

12. Karel van Mander (1604, chap. VIII), cité dans Peter Schattborn (1994 : 51).

13. Les commandes royales concernent peu en effet le paysage.

14. Ces œuvres sont bien connues grâce aux commentaires du Salon, à la correspondance entre l'artiste et Marigny publiée par Jules Guiffrey (1893), à l'étude de Laurent Manœuvre et Éric Reith (1994) et aux nombreux articles consacrés aux différentes villes portuaires par des spécialistes d'histoire de la marine dans la revue Neptunia entre 1971 et 1975.

15. Pour les vues de Paris, voir Prosper Dorbec (1908) et pour les portraits de ville en France, l'article de Jean Boutier (2014).

16. C'est probablement le succès remporté par la commande royale de quatre vues pour le château de La Muette, qui détermina la surintendance à ordonner au peintre ces quatre tableaux, pour lesquels il fut bien rétribué. Voir Jean Boutier (2014 : 26-27).

17. Voir à ce sujet Jean de Cayeux (1997), en particulier le chapitre intitulé " Peindre la ville » (73-88). À ces noms il faudrait ajouter celui de Jean-Baptiste Oudry (1686-1755), qui réalisa dès 1718 une vue du Petit Pont après l'incendie (Paris, musée Carnavalet), la seule vue de Paris de l'artiste qui nous soit connue.

18. Une exposition lui a récemment rendu justice : Le témoin méconnu : Pierre-Antoine Demachy (2014). 
19. La Surintendance des Bâtiments du Roi fut créée sous Henri IV et ses compétences s'étendent en 1664 aux manufactures de tapisseries, à l'Imprimerie royale et au Jardin royal. L'Académie royale de peinture et de sculpture fut fondée en 1648, celle des sciences en 1666, celle d'architecture en 1671 et celle de marine en 1752.

20. Comme nous l'a fait remarquer Jean Boutier, le succès d'Hubert Robert s'explique d'ailleurs surtout par la prééminence qu'il accorde à l'invention et par la forte présence des «caprices » dans son œuvre.

21. Communication écrite [courriel], 24 septembre 2015.

22. Sentiment prononcé d'appartenance à une ville.

23. Jean Boutier, communication écrite [courriel], 24 septembre 2015.

\section{RÉSUMÉS}

La vue ou veduta, sous-genre du paysage, a connu en Europe au XviII siècle un succès considérable, en particulier dans le cadre du marché du Grand Tour. La France, qui s'est pourtant brillamment illustrée depuis le $\mathrm{XVII}^{\mathrm{e}}$ siècle dans la peinture de paysage et qui compte au XVIII siècle de célèbres représentants du genre tels que Joseph Vernet et Hubert Robert, semble en revanche avoir boudé ce type de production. L'article tente de définir la peinture de paysage qui se pratique en France au Siècle des lumières et d'analyser les raisons de l'insuccès de la vue topographique.

Views or vedutes, a subgenre of landscape painting, were extremely popular in eighteenthcentury Europe, especially within the context of the Grand Tour. However, vedutes seem to have been a neglected genre in France, even though French landscape painting had flourished since the seventeenth century with such renowned painters as Joseph Vernet and Hubert Robert. The essay attempts to define landscape painting in Enlightenment France and analyze the reasons for the failure of the veduta.

\section{INDEX}

Keywords : view, veduta, France, painting, landscape, eighteenth century, theories of art Mots-clés : vue, veduta, France, peinture, paysage, dix-huitième siècle, théories artistiques

\section{AUTEUR}

\section{ÉMILIE BECK-SAIELLO}

Université Paris 13, Sorbonne Paris Cité, Pléiade (EA 7338) 